\title{
DEGENERATION OF HODGE STRUCTURES OVER PICARD MODULAR SURFACES
}

\author{
GIUSEPPE ANCONA
}

\begin{abstract}
We study variations of Hodge structures over a Picard modular surface, and compute the weights and types of their degenerations through the cusps of the Baily-Borel compactification. These computations are one of the key inputs which allow Wildeshaus [17 to construct motives associated with Picard modular forms.
\end{abstract}

Keywords: Picard surfaces; Shimura varieties; Hodge structures; motives; modular forms.

Mathematics Subject Classification 2010: 11F55, 11F75, 11G15, 14D07, 14J10.

\section{INTRODUCTION}

1.1. In [9], Scholl constructed motives associated with modular forms, lifting Deligne's construction of Galois representations attached to modular forms 4]. This is done by studying the cohomology and the motive of modular curves, together with the universal family of elliptic curves over them.

The Galois representations constructed by Deligne (as well as the motives constructed by Scholl) are pure. This is essential, for example, for Deligne's proof of Ramanujan's conjecture on the $\tau$-function [4, Theorem 5.6]. On the other hand these modular forms live in the cohomology of varieties that are smooth but not compact. Hence to understand purity one has to deal with the boundary cohomology.

A program, initiated by Wildeshaus in [14], aims to generalize such constructions to all PEL Shimura varieties. Our contribution here is to compute the boundary cohomology in the case of Picard modular surfaces. This is the key ingredient that allows Wildeshaus to construct motives associated with Picard automorphic forms [17, Theorem 3.8 and Theorem 5.6] (see also Remark [1.7).

1.2. Picard modular surfaces are the moduli spaces of abelian varieties of dimension 3 endowed with an action of an order of an imaginary quadratic field (as well as some other additional structures).

Fix a Picard modular surface $S$ and let $S^{*}$ be its Baily-Borel compactification (the boundary consists of a finite number of points). Consider $j: S \hookrightarrow S^{*}$ the canonical open immersion and $i: \mathrm{pt} \hookrightarrow S^{*}$ the inclusion of one fixed point of the boundary. Let $A$ be the universal abelian scheme over $S$ (which is of relative dimension 3), and take the $r$-fold fiber product $f: A^{r} \longrightarrow S$.

Theorem 1.3. With the above notations the following holds:

(1) the $\mathbb{Q}$-Hodge structure $i^{*} R^{0} j_{*} R^{p} f_{*} \mathbb{Q}_{A^{r}}$ has weights $\{p-t\}_{c_{p} \leq t \leq C_{p}}$,

(2) the $\mathbb{Q}$-Hodge structure $i^{*} R^{1} j_{*} R^{p} f_{*} \mathbb{Q}_{A^{r}}$ has weights $\{p+1-t\}_{0 \leq t \leq M_{p}}$, 
(3) the $\mathbb{Q}$-Hodge structure $i^{*} R^{2} j_{*} R^{p} f_{*} \mathbb{Q}_{A^{r}}$ has weights $\{p+3+t\}_{0 \leq t \leq M_{p}}$,

(4) the $\mathbb{Q}$-Hodge structure $i^{*} R^{3} j_{*} R^{p} f_{*} \mathbb{Q}_{A^{r}}$ has weights $\{p+4+t\}_{c_{p} \leq t \leq C_{p}}$,

(5) the $\mathbb{Q}$-Hodge structure $i^{*} R^{k} j_{*} R^{p} f_{*} \mathbb{Q}_{A^{r}}$ vanishes for $k \geq 4$, where

$$
\begin{aligned}
c_{p} & = \begin{cases}1 \quad \text { for } \quad p=1,6 r-1 \\
0 \text { otherwise }\end{cases} \\
C_{p} & =\min \{p, 2 r,|6 r-p|\} \\
M_{p} & =\left\{\begin{array}{lll}
p & \text { for } & p \leq r \\
r+\left[\frac{p-r}{2}\right] & \text { for } & r<p \leq 3 r \\
r+\left[\frac{5 r-p}{2}\right] & \text { for } & 3 r<p \leq 5 r \\
6 r-p & \text { for } & 5 r<p \leq 6 r
\end{array}\right.
\end{aligned}
$$

Note that the theorem above deals with the range $p \leq 6 r$ as for $p>6 r$ the sheaf $R^{p} f_{*} \mathbb{Q}_{A^{r}}$ vanishes.

1.4. Having in mind the application to modular forms explained in \$1.1 we have to deal, not only with the whole relative cohomology of the universal abelian scheme, but also with interesting direct factors. To do so, we consider the "canonical construction" functor (\$4.1)

$$
\mu_{S}: \operatorname{Rep}_{G, \mathbb{Q}} \longrightarrow \operatorname{VHS}(S)_{\mathbb{Q}},
$$

from $\mathbb{Q}$-representations of the group $G$ of the Shimura datum underlying $S$ to (admissible) variations of $\mathbb{Q}$-Hodge structures over $S$. The main result of this paper is the computation of the weights of the $\mathbb{Q}$-Hodge structure

$$
i^{*} R^{k} j_{*} \mu_{S}(F),
$$

for all integers $k$ and all $G$-representations $F$.

To compute these weights one can extend the scalars from $\mathbb{Q}$ to $\mathbb{C}$. Then, as the functors $i^{*} R^{k} j_{*} \mu_{S, \mathbb{C}}$ are linear, it is enough to consider irreducible representations of $G_{\mathbb{C}}$. In our case, there is an isomorphism of $\mathbb{C}$-algebraic groups $G_{\mathbb{C}} \cong \mathrm{GL}_{3} \times \mathbb{G}_{m}$, so that irreducible representations correspond to lists of four integers $(a, b, c, d)$ such that $a \geq b \geq c$ (such a list is called maximal weight of the representation).

The following is our main result (see Theorem 4.11 in the text) and we will deduce Theorem 1.3 from it.

Theorem 1.5. Let $F_{\lambda}$ be the irreducible representation of $G_{\mathbb{C}}$ of maximal weight $\lambda=(a, b, c, d)$. Then the following holds:

(1) $i^{*} R^{0} j_{*} \mu_{S, \mathbb{C}}\left(F_{\lambda}\right)$ is of weight $-2 a-b-2 d$,

(2) $i^{*} R^{1} j_{*} \mu_{S, \mathbb{C}}\left(F_{\lambda}\right)$ has weights $-a-2 b-2 d+1$ and $-2 a-c-2 d+1$,

(3) $i^{*} R^{2} j_{*} \mu_{S, \mathbb{C}}\left(F_{\lambda}\right)$ has weights $-a-2 c-2 d+3$ and $-2 b-c-2 d+3$,

(4) $i^{*} R^{3} j_{*} \mu_{S, \mathbb{C}}\left(F_{\lambda}\right)$ is of weight $-b-2 c-2 d+4$,

(5) $i^{*} R^{k} j_{*} \mu_{S, \mathbb{C}}\left(F_{\lambda}\right)$ vanishes for $k \geq 4$.

Corollary 1.6. Let us keep the notations from the theorem above and write $w$ for the weight of the variation of pure Hodge structures $\mu_{S, \mathbb{C}}\left(F_{a, b, c, d}\right)$. Suppose that $F_{\lambda}$ is regular (i.e. $a \neq b \neq c$ ). Then, for each non-negative integer $k$, the integers $k+w$ and $k+w+1$ are not weights of $i^{*} R^{k} j_{*} \mu_{S, \mathbb{C}}\left(F_{\lambda}\right)$. 
Remark 1.7. Let us explain in more details the connection of Corollary 1.6] with the construction of motives associated with modular forms (the reader can alternatively consult the introduction of [17]).

Let $S=S h^{K}(G, X)$ be a PEL Shimura variety and $A$ the universal abelian scheme over it. By [1, Theorem 8.6], for any $G$-representation $F_{\lambda}$, the variation of Hodge structures $\mu_{S}\left(F_{\lambda}\right)$ over $S$ is the realization of a canonical relative Chow motive over $S$ of the form $M_{S}\left(A^{r}, p, s\right)$ for some integers $r$ and $s$. Here $A^{r}$ is the $r$-fold product of $A$ over $S, p$ is a projector and, $(s)$ is the Tate twist.

By [15, Theorem 2.2], the projector $p$ induces a direct factor $M\left(A^{r}\right)^{p}$ of the mixed motive $M\left(A^{r}\right)$ of the smooth and non-projective variety $A^{r}$, as well as a direct factor $\partial M\left(A^{r}\right)^{p}$ of the boundary motive $\partial M\left(A^{r}\right)$.

The mixed motive $M\left(A^{r}\right)^{p}$ is endowed with an action of the Hecke algebra. In order to construct motives associated with modular forms one would like to have a pure motive which is the best "pure approximation" of $M\left(A^{r}\right)^{p}$ and which is still endowed with an action of the Hecke algebra, then use its action to cut pure smaller parts.

By [14, Theorem 4.3], this is possible if the boundary motive $\partial M\left(A^{r}\right)^{p}(s)$ avoids weights -1 and 0 . This weight condition however cannot be satisfied by all $F_{\lambda}$. Clearly, a necessary condition is to check this on realizations. By [16, Theorem 1.2, Proposition 3.5] this corresponds to the fact that, for each non-negative integer $k$, the integers $k+\bar{w}$ and $k+\bar{w}+1$ are not weights of $i^{*} R^{k} j_{*} \mu_{S, \mathbb{C}}\left(F_{\lambda}\right)$ (with $\bar{w}$ being the weight of $\left.\mu_{S, \mathbb{C}}\left(F_{\lambda}\right)\right)$. In the case of Picard surfaces, Corollary 1.6 shows that this condition is satisfied for all regular representations.

Again in the Picard case, it is shown in [17 that this control of weights in cohomology is actually sufficient to control the motivic weights (i.e., for Picard surfaces, conservativity of the realization can be shown, see [17. Theorem 3.8]). In particular, one can deduce canonical pure motives associated with regular representations [17, Corollary 3.9], and from them, by using the action of the Hecke algebra, one can cut out motives attached to Picard automorphic forms [17, Theorem 5.6].

1.8. The main tool in the proof of Theorem 1.5 is a theorem of Burgos and Wildeshaus [3], which works for a general Shimura variety and reduces the computation of weights of degenerations to a computation of cohomology of groups. In our special case the groups involved are concrete enough to allow us to explicitly perform all the required computations.

Note that one could alternatively have used [6, Theorem 4.3.14] instead of [3] as starting point for our computations.

1.9. Organization of the paper. In Section 2 we recall generalities about Picard surfaces and their Shimura datum. The standard reference is [5].

In Section 3 we describe the boundary of Baily-Borel and toroidal compactifications, as well as the Shimura datum underlying the strata of these compactifications.

Section 4 contains the main results concerning the degeneration of Hodge structures.

1.10. Notations and convention. We will write $\mathbb{A}_{f}$ for the ring of finite adeles. We will write $\mathbb{S}=\operatorname{Res}_{\mathbb{C} / \mathbb{R}} \mathbb{G}_{m, \mathbb{C}}$ for the $\mathbb{R}$-algebraic group called Deligne's torus. Here $\operatorname{Res}_{\mathbb{C} / \mathbb{R}}$ is the Weil restriction of scalars. 
When $L \supset K$ are fields and $X$ is an object defined over $K$ (for instance a variety or a group), we will write $X_{L}$ for its base change to $L$.

\section{PiCARD DATUM}

In this section we recall the definition of a Picard datum $(G, X)$; the standard reference is [5]. This is a Shimura datum of PEL type. We first construct the $\mathbb{Q}$-algebraic group $G$, which after extending scalars becomes the direct product of a linear group by the multiplicative group (Remark 2.3).

2.1. Notation. Let $E$ an imaginary quadratic field embedded in $\mathbb{C}$, and $n$ the only positive square-free integer such that

$$
E=\mathbb{Q}(i \sqrt{n}) .
$$

Let $V=V_{E}$ be an $E$-vector space of dimension 3, and $J$ be a hermitian form of signature $(2,1)$. In particular, one can find three orthogonal vectors $v_{1}, v_{2}, v_{3} \in V$ such that $J\left(v_{1}, v_{1}\right)$ and $J\left(v_{2}, v_{2}\right)$ are positive and $J\left(v_{3}, v_{3}\right)$ is negative.

Definition 2.2. In the context of Notation 2.1 we define $G=G U(V, J)$ to be the $\mathbb{Q}$-algebraic group of the $E$-linear automorphisms of $V$ which respect the hermitian form $J$ up to scalar.

Remark 2.3. Let $V_{\mathbb{Q}}$ be $V$ viewed as a $\mathbb{Q}$-vector space. Then the algebra $E \otimes E$ acts on the $E$-vector space $V_{\mathbb{Q}} \otimes_{\mathbb{Q}} E$, and hence induces a decomposition

$$
V_{\mathbb{Q}} \otimes_{\mathbb{Q}} E=V_{i} \oplus V_{-i},
$$

where $\quad V_{i}=\left\{w \in V_{\mathbb{Q}} \otimes_{\mathbb{Q}} E, \quad(a \otimes 1) w=(1 \otimes a) w \forall a \in E\right\}$,

and $\quad V_{-i}=\left\{w \in V_{\mathbb{Q}} \otimes_{\mathbb{Q}} E, \quad(a \otimes 1) w=(1 \otimes \bar{a}) w \quad \forall a \in E\right\}$.

Let $\mathcal{B}=\left\{v_{1}, v_{2}, v_{3}\right\}$ be an $E$-basis of $V$; and write $\pi_{i}: V_{\mathbb{Q}} \otimes_{\mathbb{Q}} E \longrightarrow V_{i}$ for the projector whose kernel is $V_{-i}$. Then

$$
\mathcal{B}_{i}=\left\{\pi_{i}\left(v_{1}\right), \pi_{i}\left(v_{2}\right), \pi_{i}\left(v_{3}\right)\right\}
$$

is a basis of $V_{i}$ and one has an isomorphism

$$
\phi_{\mathcal{B}}: G_{E} \stackrel{\sim}{\longrightarrow} \mathrm{GL}_{3, E} \times \mathbb{G}_{m, E}
$$

given by $g \mapsto\left(g_{\mid V_{i}}, \chi_{g}\right)$, where the restriction $g_{\mid V_{i}}$ of $g$ to $V_{i}$ is written in the basis $\mathcal{B}_{i}$ and $\chi_{g}$ is the scalar such that $J(g \cdot, g \cdot)=\chi_{g} J(\cdot, \cdot)$.

2.4. Convention. Let $\mathcal{B}=\left\{v_{1}, v_{2}, v_{3}\right\}$ be an $E$-basis of $V$. Then

$$
\widetilde{\mathcal{B}}=\left\{v_{1}, i \sqrt{n} v_{1}, v_{2}, i \sqrt{n} v_{2}, v_{3}, i \sqrt{n} v_{3}\right\}
$$

is a $\mathbb{Q}$-basis of $V$. For any $\mathbb{Q}$-algebra $R$, and any $R$-point $g \in G(R)$, we will write

$$
g=\left(\begin{array}{lll}
\left(a_{11}, b_{11}\right) & \left(a_{12}, b_{12}\right) & \left(a_{13}, b_{13}\right) \\
\left(a_{21}, b_{21}\right) & \left(a_{22}, b_{22}\right) & \left(a_{23}, b_{23}\right) \\
\left(a_{31}, b_{31}\right) & \left(a_{32}, b_{32}\right) & \left(a_{33}, b_{33}\right)
\end{array}\right),
$$

where $a_{j k} \in R$ is the coordinate of $g\left(v_{k}\right)$ with respect to $v_{j}$ and $b_{j k} \in R$ is the coordinate of $g\left(v_{k}\right)$ with respect to $i \sqrt{n} v_{j}$. Note that, by definition of $G$, we have $g\left(i \sqrt{n} v_{k}\right)=i \sqrt{n} g\left(v_{k}\right)$, in particular the $a_{j k} \in R$ and $b_{j k} \in R$ determine $g$. 
Definition 2.5. Let $\mathcal{B}=\left\{v_{1}, v_{2}, v_{3}\right\}$ be an $E$-basis of $V$ such that $J\left(v_{1}, v_{1}\right)$ and $J\left(v_{2}, v_{2}\right)$ are positive and $J\left(v_{3}, v_{3}\right)$ is negative (see Notation 2.1). Consider the morphism of algebraic groups $h_{v_{1}, v_{2}, v_{3}}: \mathbb{S} \longrightarrow G_{\mathbb{R}}$ given by

$$
h_{v_{1}, v_{2}, v_{3}}:\left(z_{1}, z_{2}\right) \mapsto\left(\begin{array}{ccc}
\left(\frac{z_{1}+z_{2}}{2}, \frac{z_{1}-z_{2}}{2 i \sqrt{n}}\right) & (0,0) & (0,0) \\
(0,0) & \left(\frac{z_{1}+z_{2}}{2}, \frac{z_{1}-z_{2}}{2 i \sqrt{n}}\right) & (0,0) \\
(0,0) & (0,0) & \left(\frac{z_{1}+z_{2}}{2}, \frac{z_{2}-z_{1}}{2 i \sqrt{n}}\right)
\end{array}\right),
$$

where $G$ is the group of Definition 2.2 and the morphism is written in the basis $\mathcal{B}$ using Convention [2.4. We will write $X$ for the topological space of the $G(\mathbb{R})$ conjugacy class of the morphism $h_{v_{1}, v_{2}, v_{3}}$.

Definition 2.6. The pair $(G, X)$ will be called a Picard datum. When $K \subset G\left(\mathbb{A}_{f}\right)$ is a neat subgroup [8, §0.5] we will write $S=S h^{K}(G, X)$ for the induced Shimura variety. It is a complex], smooth and quasi-projective surface which we call a "Picard modular surface"; see [5] for details and proofs.

Remark 2.7. The Picard modular surface $S$ is the fine moduli space of polarised abelian varieties of dimension 3 endowed with an action of an order of $E$ and some additional structures (depending also on $K$ ). In particular, there is a universal abelian scheme $f: A \rightarrow S$; see [5] for details and proofs.

\section{COMPACTIFICATIONS AND BOUNDARY}

The aim of this section is to describe the Shimura data underlying the strata of the boundary of the Baily-Borel and toroidal compactifications of a Picard modular surface $S$ (Definition 2.6). These strata are associated to parabolic subgroups of the group $G$ introduced in Definition 2.2 (for generalities on strata of compactifications of Shimura varieties see [8, Chapter 4]). We start by describing these parabolic subgroups (3.1]3.3), then the Shimura datum associated to each stratum (3.4][3.10) and deduce the geometry of the boundary (3.12) 3.14).

Lemma 3.1. Let $(V, J)$ be as in Notation 2.1. Then there exist infinitely many isotropic vectors in $V$. Moreover if $\widetilde{v}$ be any non-zero isotropic vector, then there exists a positive rational number $b$ and an isomorphism $(V, J) \stackrel{\sim}{\longrightarrow}\left(E^{3}, J_{b}\right)$ sending $\widetilde{v}$ to the first vector of the canonical base of $E^{3}$. Here $J_{b}$ is the hermitian form

$$
J_{b}=\left(\begin{array}{ccc}
0 & 0 & 1 \\
0 & b & 0 \\
1 & 0 & 0
\end{array}\right)
$$

Proof. For the first part, let us diagonalize the hermitian form $J$. Then we have to look for rational solutions of an equation of the form $\sum_{i=1}^{6} c_{i} t_{i}^{2}=0$ with $c_{i}$ integers, four positive and two negative. This indeed has a solution (and thus infinitely many) by [10, corollaire 2, p. 77, chap. 4]. The rest is standard.

Definition 3.2. Let $D$ be an isotropic $E$-line of $V$. An $E$-basis $w_{1}, w_{2}, w_{3}$ of $V$ is called a parabolic basis adapted to $D$ if $w_{1}$ generates $D$, and the matrix representing $J$ in this basis is of the form $J_{b}$ for some $b$ (following notations of Lemma 3.1).

\footnotetext{
${ }^{1}$ In fact, it has a (canonical) model over the imaginary quadratic field $E[\underline{5}]$.
} 
Proposition 3.3. Let $D$ be an isotropic E-line of $V$ (see Lemma 3.1), and define $Q_{D}$ to be the subgroup of $G$ stabilizing $D$. Then

$$
Q_{D}=G \cap\left\{\left(\begin{array}{ccc}
\left(a_{11}, b_{11}\right) & \left(a_{12}, b_{12}\right) & \left(a_{13}, b_{13}\right) \\
(0,0) & \left(a_{22}, b_{22}\right) & \left(a_{23}, b_{23}\right) \\
(0,0) & (0,0) & \left(a_{33}, b_{33}\right)
\end{array}\right)\right\}
$$

where the coordinates are written using Convention 2.4 and we are using a parabolic basis adapted to $D$ (Definition 3.2). The unipotent radical of $Q_{D}$ is

$$
R_{u}\left(Q_{D}\right)=\left\{\left(\begin{array}{ccc}
(1,0) & \left(-b a_{23}, b b_{23}\right) & \left(-\frac{b}{2}\left(a_{23}^{2}+n b_{23}^{2}\right), b_{13}\right) \\
(0,0) & (1,0) & \left(a_{23}, b_{23}\right) \\
(0,0) & (0,0) & (1,0)
\end{array}\right)\right\},
$$

with Lie algebra

$$
\text { Lie } R_{u}\left(Q_{D}\right)=\left\{\left(\begin{array}{ccc}
(0,0) & \left(-b a_{23}, b b_{23}\right) & \left(0, b_{13}\right) \\
(0,0) & (0,0) & \left(a_{23}, b_{23}\right) \\
(0,0) & (0,0) & (0,0)
\end{array}\right)\right\} .
$$

The torus

$$
T_{m, D}=\left\{\left(\begin{array}{ccc}
\left(\frac{\lambda_{1}+\lambda_{2}}{2}, \frac{\lambda_{1}-\lambda_{2}}{2 i \sqrt{n}}\right) & (0,0) & (0,0) \\
(0,0) & \left(\frac{\lambda_{3}+\lambda_{4}}{2}, \frac{\lambda_{3}-\lambda_{4}}{2 i \sqrt{n}}\right) & (0,0) \\
(0,0) & (0,0) & \left(\frac{\frac{\lambda_{3} \lambda_{4}}{\lambda_{2}}+\frac{\lambda_{3} \lambda_{4}}{\lambda_{1}}}{2}, \frac{\frac{\lambda_{3} \lambda_{4}}{\lambda_{2}}-\frac{\lambda_{3} \lambda_{4}}{\lambda_{1}}}{2 i \sqrt{n}}\right)
\end{array}\right)\right\}
$$

is a maximal torus of $G$ defined over $\mathbb{C}$, and the torus

$$
T_{D}=\left\{\mu \cdot\left(\begin{array}{ccc}
(\lambda, 0) & (0,0) & (0,0) \\
(0,0) & (1,0) & (0,0) \\
(0,0) & (0,0) & \left(\lambda^{-1}, 0\right)
\end{array}\right)\right\}
$$

is a maximal split torus defined over $\mathbb{Q}$. There is only one Borel subgroup $B_{D}$ of $G$ such that $Q_{D} \supseteq B_{D} \supseteq T_{D}$ and it is $Q_{D}$ itself.

Moreover $Q_{D}$ is an admissible parabolic subgroup of $G$ in the sense of 8 , Definition 4.5] and the admissible parabolic subgroups are exactly those subgroups of the form $Q_{D^{\prime}}$ for some isotropic E-line $D^{\prime}$ of $V$.

Proof. Let $w_{1}, w_{2}, w_{3}$ be a parabolic basis adapted to $D$. The group $Q_{D}$ stabilizes the line $D$ and so it has to stabilize also $D^{\perp}$ the plan orthogonal to $D$. As $D$ is generated by $w_{1}$ and $D^{\perp}$ is generated by $w_{1}$ and $w_{2}$ we deduce the description of $Q_{D}$ in the statement. The unipotent radical of $Q_{D}$ is

$$
R_{u} Q_{D}=G \cap\left\{\left(\begin{array}{ccc}
(1,0) & \left(a_{12}, b_{12}\right) & \left(a_{13}, b_{13}\right) \\
(0,0) & (1,0) & \left(a_{23}, b_{23}\right) \\
(0,0) & (0,0) & (1,0)
\end{array}\right)\right\} .
$$

By imposing the condition of being elements of the group $G$, we find the equations in the statement.

A computation shows that the elements of $T_{m, D}$ belong to $G$ and hence to $Q_{D}$. This torus is of dimension 4 and so, by Remark 2.3, it is maximal.

All maximal torus over $\mathbb{C}$ are conjugated, in particular on the diagonal of any such torus $T$ we will have the same coordinates appearing in $T_{m, D}$. In particular, a subtorus of $T$ that is defined and splits over $\mathbb{Q}$ must verify $\lambda_{1}=\lambda_{2}$ and $\lambda_{3}=\lambda_{4}$, hence it is of dimension at most 2. As $T_{D}$ has dimension 2, it is a maximal split torus defined over $\mathbb{Q}$. 
Note that by the description of $Q_{D}, R_{u} Q_{D}$ and $T_{m, D}$ we gave above, we have $Q_{D} / R_{u} Q_{D} \cong T_{m, D}$. In particular $Q_{D}$ has dimension 7 (and it is connected). By Remark 2.3, $B_{D}$ must have dimension 7, hence $B_{D}=Q_{D}$.

By Remark 2.3 the adjoint group of $G$ is simple up to isogeny (namely it is a $\mathbb{Q}$-form of $\mathrm{SL}_{3}$ ), in particular the admissible parabolic subgroups of $G$ are the maximal $\mathbb{Q}$-parabolic subgroups of $G$. It is clear that a subgroup of the form $Q_{D^{\prime}}$ is a parabolic subgroup. Let us show now that any $\mathbb{Q}$-parabolic subgroup of $G$ is contained in one subgroup of the form $Q_{D^{\prime}}$. Following notations from Remark 2.3] a parabolic subgroup $P$ defined over $E$ has to stabilize a line of $V_{i}$; moreover it is of dimension at least 7 . If $P$ is moreover defined over $\mathbb{Q}$, then it has to stabilize a line $l$ of $V$, and hence also the orthogonal plan $l^{\perp}$. This line has to be isotropic, otherwise these two conditions force $P$ to be of dimension at most 6 .

Lemma 3.4. Let $\left(Q_{D}, B_{D}, T_{D}\right)$ be as in Proposition 3.3. Consider the cocharacter $\lambda_{D}: \mathbb{G}_{m, \mathbb{Q}} \longrightarrow T_{D}$ given in a parabolic basis adapted to $D$ (Definition 3.2 ) by

$$
\lambda_{D}: t \mapsto\left\{\left(\begin{array}{ccc}
(t, 0) & (0,0) & (0,0) \\
(0,0) & (1,0) & (0,0) \\
(0,0) & (0,0) & \left(t^{-1}, 0\right)
\end{array}\right)\right\}
$$

(we write coordinates using Convention 2.4). Then $\lambda_{D}$ is the cocharacter associated to the data $\left(Q_{D}, B_{D}, T_{D}\right)$ in the general formalism of $[8, \S 4.1]$.

Proof. Any cocharacter $\lambda: \mathbb{G}_{m, \mathbb{Q}} \longrightarrow T_{D}$ is of the form

$$
t \mapsto\left\{t^{b}\left(\begin{array}{ccc}
\left(t^{a}, 0\right) & (0,0) & (0,0) \\
(0,0) & (1,0) & (0,0) \\
(0,0) & (0,0) & \left(t^{-a}, 0\right)
\end{array}\right)\right\}
$$

By [8, §4.1], the image of $\lambda_{D}$ has to be contained in the derived group of $G$, so $b=0$.

Consider the action of $\mathbb{G}_{m, \mathbb{Q}}$ over Lie $G$ induced by $\lambda_{D}$. By $[8, \S 4.1]$, the subLie algebra $\operatorname{Lie} Q_{D} \subset$ Lie $G$ coincide with the sum of the eigenspaces associated to eigenvalues of non-negative weights, hence $a \geq 0$. Note that in the decomposition Lie $Q_{D}=(\operatorname{Lie} G)_{0}+(\operatorname{Lie} G)_{a}+(\operatorname{Lie} G)_{2 a}$ each of the three eigenspaces is non-trivial. Also note that we have $\operatorname{Lie} R_{u}\left(Q_{D}\right)=(\operatorname{Lie} G)_{a}+(\operatorname{Lie} G)_{2 a}$.

On the other hand, as $G$ is reductive, $R_{u}\left(Q_{D}\right)$ is the unipotent radical of a group belonging to a Shimura datum (it will be the group $P_{D}$ of Lemma 3.8) and the decomposition $\operatorname{Lie} R_{u}\left(Q_{D}\right)=(\operatorname{Lie} G)_{a}+(\operatorname{Lie} G)_{2 a}$ is the one induced by the Shimura datum (see $[8, \S 4.8,4.9,4.10]$ ). In particular, as the weights allowed in a mixed Shimura datum are $0,-1$ et -2 (see [8, Definition 2.1]), we must have $a=1$.

3.5. Notation. Following $[8, \S 4.2,4.3]$, we write the following morphisms of algebraic groups $h_{0}: \mathbb{S}_{\mathbb{C}} \rightarrow \mathbb{S}_{\mathbb{C}} \times \mathrm{GL}_{2, \mathbb{C}}$

$$
h_{0}:\left(z_{1}, z_{2}\right) \mapsto\left[\left(z_{1}, z_{2}\right),\left(\begin{array}{cc}
\frac{z_{1}+z_{2}}{2} & \frac{z_{2}-z_{1}}{2 i} \\
\frac{z_{1}-z_{2}}{2 i} & \frac{z_{1}+z_{2}}{2}
\end{array}\right)\right],
$$


and $h_{\infty}: \mathbb{S}_{\mathbb{C}} \rightarrow \mathbb{S}_{\mathbb{C}} \times \mathrm{GL}_{2, \mathbb{C}}$

$$
h_{\infty}:\left(z_{1}, z_{2}\right) \mapsto\left[\left(z_{1}, z_{2}\right),\left(\begin{array}{cc}
1 & i\left(z_{1} z_{2}-1\right) \\
0 & z_{1} z_{2}
\end{array}\right)\right] .
$$

We will consider also the "weight morphism" $p: \mathbb{G}_{m,,_{R}} \rightarrow \mathbb{S}$ given by $z \mapsto(z, z)$.

Lemma 3.6. Let $D$ be an isotropic $E$-line of $V$, and $\mathcal{B}=\left\{w_{1}, w_{2}, w_{3}\right\}$ a parabolic basis adapted to $D$ (Definition 3.2). Consider the map $\omega_{w_{1}, w_{2}, w_{3}}: \mathbb{S}_{\mathbb{C}} \times \mathrm{GL}_{2, \mathbb{C}} \rightarrow G_{\mathbb{C}}$ given (in the basis $\mathcal{B}$ and using Convention 2.4) by

$$
\left[\left(z_{1}, z_{2}\right),\left(\begin{array}{cc}
a & b \\
c & d
\end{array}\right)\right] \mapsto\left(\begin{array}{ccc}
(d, 0) & (0,0) & \left(0, \frac{-b}{\sqrt{n}}\right) \\
(0,0) & \left(\frac{z_{1}+z_{2}}{2}, \frac{z_{1}-z_{2}}{2 i \sqrt{n}}\right) & (0,0) \\
\left(0, \frac{c}{\sqrt{n}}\right) & (0,0) & (a, 0)
\end{array}\right)
$$

Then $\omega_{w_{1}, w_{2}, w_{3}}$ is the only map verifying the following properties:

- it is a morphism of algebraic groups defined over $\mathbb{R}$;

- the equality $\omega_{w_{1}, w_{2}, w_{3}} \circ h_{0}=h_{w_{2}, w_{1}+w_{3}, w_{1}-w_{3}}$ holds,

- the cocharacters $\lambda_{D} \cdot\left(h_{w_{2}, w_{1}+w_{3}, w_{1}-w_{3}} \circ p\right)$ and $\omega_{w_{1}, w_{2}, w_{3}} \circ h_{\infty} \circ p$ are conjugated one to the other by an element of $Q_{D}(\mathbb{C})$.

Here the morphism $h_{w_{2}, w_{1}+w_{3}, w_{1}-w_{3}}$ is defined in Definition 2.5, the group $Q_{D}$ is defined in Proposition 3.3. the cocharacter $\lambda_{D}$ is defined in Lemma 3.4. and the morphisms $h_{0}, h_{\infty}, p$ are defined in Notation 3.5 .

Proof. Existence and uniqueness of such a morphism come from 8, Proposition 4.6]. The properties are easy to check. Note that the cocharacters

$$
t \mapsto\left(\begin{array}{ccc}
\left(t^{2}, 0\right) & (0,0) & (0,0) \\
(0,0) & (t, 0) & (0,0) \\
(0,0) & (0,0) & (1,0)
\end{array}\right) \text { and } t \mapsto\left(\begin{array}{ccc}
\left(t^{2}, 0\right) & (0,0) & \left(0, \frac{i\left(1-t^{2}\right)}{\sqrt{n}}\right) \\
(0,0) & (t, 0) & (0,0) \\
(0,0) & (0,0) & (1,0)
\end{array}\right)
$$

are conjugated one to the other by

$$
\left(\begin{array}{ccc}
(1,0) & (0,0) & \left(0,-\frac{i}{\sqrt{n}}\right) \\
(0,0) & (1,0) & (0,0) \\
(0,0) & (0,0) & (1,0)
\end{array}\right)
$$

which belongs to $Q_{D}(\mathbb{C})$.

Definition 3.7. Let us keep the notation from Lemma 3.6, we will write

$$
h_{\mathcal{B}, \infty}: \mathbb{S}_{\mathbb{C}} \rightarrow Q_{D, \mathbb{C}}
$$

for the morphism $\omega_{w_{1}, w_{2}, w_{3}} \circ h_{\infty}$; explicitly

$$
\left(z_{1}, z_{2}\right) \mapsto\left(\begin{array}{ccc}
\left(z_{1} z_{2}, 0\right) & (0,0) & \left(0, \frac{i\left(1-z_{1} z_{2}\right)}{\sqrt{n}}\right) \\
& \left(\frac{z_{1}+z_{2}}{2}, \frac{z_{1}-z_{2}}{2 i \sqrt{n}}\right) & (0,0) \\
& & (1,0)
\end{array}\right)
$$


Lemma 3.8. The smallest normal $\mathbb{Q}$-subgroup of the group $Q_{D}$ (Proposition 3.3) containing the image of the morphism $h_{\mathcal{B}, \infty}$ (Definition 3.7) is

$P_{D}=G \cap\left\{\left(\begin{array}{ccc}\left(z_{1} z_{2}, 0\right) & * & * \\ & \left(\frac{z_{1}+z_{2}}{2}, \frac{z_{1}-z_{2}}{2 i \sqrt{n}}\right) & * \\ & & (1,0)\end{array}\right)\right\}=G \cap\left\{\left(\begin{array}{ccc}\left(a^{2}+b^{2}, 0\right) & * & * \\ & \left(a, \frac{b}{\sqrt{n}}\right) & * \\ & & (1,0)\end{array}\right)\right\}$.

Moreover, $P_{D}(\mathbb{R})$ is path connected and $W_{D}$, the unipotent radical of $P_{D}$, coincides with $R_{u} Q_{D}$, the unipotent radical of $Q_{D}$.

Proof. First of all, note that the equality $W_{D}=R_{u} Q_{D}$ holds a priori by [8, proof of Lemma 4.8]. Now, the image of the morphism $h_{\mathcal{B}, \infty}$ is the group

$$
\operatorname{Im}=\left\{\left(\begin{array}{ccc}
\left(a^{2}+b^{2}, 0\right) & (0,0) & \left(0, \frac{i\left(1-a^{2}-b^{2}\right)}{\sqrt{n}}\right) \\
& \left(a, \frac{b}{\sqrt{n}}\right) & (0,0) \\
& & (1,0)
\end{array}\right)\right\} .
$$

Note that $P_{D}$ described in the statement is a normal subgroup $Q_{D}$ and it contains Im. On the other hand, the group $P_{D}$ has to contain $W_{D}$. We deduce that $P_{D}$ cannot be smaller.

Let us now show that the group $P_{D}(\mathbb{R})$ is path connected. First note that, as subgroup of $\mathrm{GL}_{3, \mathbb{C}}$, it coincides to the set of elements of the form

$$
\left\{\left(\begin{array}{ccc}
|z|^{2} & w_{1} & w_{2} \\
& z & w_{3} \\
& & 1
\end{array}\right)\right\}
$$

respecting the hermitian form $J_{b}$ up to a scalar (see Definition 3.2). In particular it is generated by the two subgroups

$$
\left\{\left(\begin{array}{ccc}
|z|^{2} & & \\
& z & \\
& & 1
\end{array}\right)\right\} \text { and }\left\{\left(\begin{array}{ccc}
1 & -b t+i b u & b \frac{t^{2}+u^{2}}{2}+i v \\
& 1 & t+i u \\
& & 1
\end{array}\right)\right\}
$$

which are both path-connected.

Definition 3.9. Let $p: \mathbb{G}_{m, R} \rightarrow \mathbb{S}$ be as in $\$ 3.5, h_{\mathcal{B}, \infty}$ as in Definition 3.7 and $P_{D}$ as in Lemma 3.8. Following [8, Lemma 4.8], we define the unipotent algebraic group $U_{D}$ and the topological space $X_{D}$ as follows. Define

$$
U_{D}=\exp \left(W_{-2} \operatorname{Lie} P_{D}\right),
$$

with $W_{-2}$ Lie $P_{D}$ being the subspace of Lie $P_{D}$ where, for $t \in \mathbb{G}_{m}$, the action of $h_{\mathcal{B}, \infty} \circ p(t)$ is given by the multiplication by $t^{2}$. Define $X_{D}$ as the orbit of $h_{\mathcal{B}, \infty}$ under the action by conjugation of $P_{D}(\mathbb{R}) U_{D}(\mathbb{C})$.

Proposition 3.10. The topological space $X_{D}$ (see Definition 3.9) is connected and the pair $\left(P_{D}, X_{D}\right)$ is a (mixed) Shimura datum (see [8, Definition 2.1]). Moreover $\left(P_{D}, X_{D}\right)$ is a proper rational boundary component of $(G, X)$, and any proper rational boundary component of $(G, X)$ is of the form $\left(P_{D^{\prime}}, X_{D^{\prime}}\right)$ for some isotropic E-line $D^{\prime}$ of $V$ (see [8, §4.11] for generalities on proper rational boundary components). 
Proof. The space $X_{D}$ is connected as $P_{D}(\mathbb{R})$ is (Lemma 3.8). The general theory 8 , $\S 4.1-4.11]$ and our previous results in this section imply that $\left(P_{D}, X_{D}\right)$ is a proper rational boundary component of $(G, X)$. As any admissible parabolic subgroup is of the form $Q_{D^{\prime}}$ (Proposition 3.3 ) then any proper rational boundary component of $(G, X)$ is of the form $\left(P_{D^{\prime}}, X_{D^{\prime}}\right)$

Lemma 3.11. The unipotent groups $W_{D}$ (Lemma 3.8) and $U_{D}$ (Definition 3.10) are of dimension respectively 3 and 1.

Proof. By Lemma 3.8, $W_{D}$ coincides with the unipotent group $R_{u} Q_{D}$. Using the description of $R_{u} Q_{D}$ in Proposition 3.3 we have that $W_{D}$ has dimension 3.

Following the proof of Lemma 3.4, we have Lie $R_{u}\left(Q_{D}\right)=(\operatorname{Lie} G)_{a}+(\operatorname{Lie} G)_{2 a}$ and Lie $U_{D}=(\operatorname{Lie} G)_{2 a}$. Then we can conclude combining this with the description of Lie $R_{u} Q_{D}$ in Proposition 3.3 .

Proposition 3.12. Let $\left(P_{D}, X_{D}\right)$ be the Shimura datum of Proposition $3.10, W_{D}$ the unipotent radical of $P_{D}$ and $U_{D}$ the unipotent group as in Definition 3.9. Consider the quotients of Shimura data (in the sense of [8, Proposition 2.9])

$$
\left(P_{D}, X_{D}\right) / W_{D}=\left(P_{D} / W_{D}, X_{D}^{1}\right) \text { and }\left(P_{D}, X_{D}\right) / U_{D}=\left(P_{D} / U_{D}, X_{D}^{2}\right) \text {. }
$$

Then, as complex analytic variety, $X_{D}^{1}$ is a point, $X_{D}^{2}$ is an affine space of dimension 1 and $X_{D}$ is an affine space of dimension 2.

Proof. Consider the unipotent quotients $P_{D} \rightarrow P_{D} / U_{D} \rightarrow P_{D} / W_{D}$. As $X_{D}$ is connected (Proposition [3.10), by [8, Remark 2.9] the topological spaces $X_{D}^{1}$ and $X_{D}^{2}$ are also connected. On the other hand $P_{D} / W_{D}$ is commutative, so $X_{D}^{1}$ is a finite number of points, hence a point.

Then, by the general results on unipotent extensions $[8, \S 2.18,2.19], X_{D}^{2}$ is a $\mathbb{C}$ vector space whose real dimension coincides with the one of $\left(W_{D} / U_{D}\right)$. We conclude using the Lemma 3.11

Again by [8, $\S 2.18,2.19], X_{D}$ is a vector bundle over $X_{D}^{2}$ whose fiber have complex dimension coinciding with the one of $U_{D}$. By Lemma 3.11 this is a line bundle, and as $X_{D}^{2}$ is contractible, the line bundle is trivial.

Corollary 3.13. Let $S$ be a Picard modular surface as defined in Definition 2.6, $\partial S$ be the boundary of the Baily-Borel compactification of $S$ and $\partial S^{T}$ be the boundary of the toroidal compactification of $S$. Then the Shimura data underlying the strata of $\partial S$ are of the form $\left(P_{D} / W_{D}, X_{D}^{1}\right)$ and the Shimura data underlying the strata $\partial S^{T}$ are of the form $\left(P_{D} / U_{D}, X_{D}^{2}\right)$. In particular, as complex varieties, $\partial S$ is a finite number $N$ of points and $\partial S^{T}$ is a disjoint union of $N$ smooth and proper curves of genus 1 .

Remark 3.14. The number $N$ in the previous proposition is computed in several cases in [11. Note also that the union of the $N$ points (or the union of the $N$ curves) is actually defined over the imaginary quadratic field $E$, but a priori each point is not. The geometry of $\partial S^{T}$ appears also in [7] and [2, Chapter 1]. 
Proof. The general theory ([8, Chapter 6] and [13, Lemma 1.7]), Proposition 3.10 and Proposition 3.12 imply the Baily-Borel case, as well as the fact that the Shimura data underlying the strata of $\partial S^{T}$ are of the form $\left(P_{D}, X_{D}\right) / U_{D}^{\sigma}$, with $U_{D}^{\sigma}$ a subgroup of the unipotent group $U_{D}$ (Definition 3.9).

If $U_{D}^{\sigma}$ were trivial, then the boundary would be of dimension 2 by Proposition 3.12, which is impossible as $S$ is a surface. On the other hand $U_{D}$ has dimension 1 by Lemma 3.11 hence $U_{D}^{\sigma}=U_{D}$.

\section{Degeneration of Hodge structures}

This section contains the main result, Theorem 4.11, We study how variations of Hodge structures over a Picard modular surface degenerate through the cusps of its Baily-Borel compactification. More precisely, we describe the types of the Hodge structures $R^{k} i^{*} j_{*} \mu_{S}(F)$ for all $G$-representations $F$ (see Notation 4.1). Remark4.2 shows that these structures have a geometric interest.

We start by reducing the problem to a combinatorial question (4.34.6). The main ingredient is a theorem of Burgos and Wildeshaus, which in this case has a simplified version via Lemma 4.3. We then deal with this combinatorial question (4.744.9) and finally describe the Hodge structures we are interested in (4.1044.12). The last part (4.134.15) explains how to deduce Theorem 1.3 and Corollary 1.6 from Theorem 4.11 .

4.1. Notation. Let $S$ be a Picard modular surface (Definition 2.6), $S^{*}$ be its Baily-Borel compactification and $j: S \hookrightarrow S^{*}$ be the canonical open immersion. By Corollary 3.13 the boundary of $S^{*}$ is a finite set of points. Let us fix one of these points, and let $i: \mathrm{pt} \hookrightarrow S^{*}$ be the inclusion.

Let $(G, X)$ be the Shimura datum underlying $S$ (Definition 2.6),

$$
\left(G_{\mathrm{pt}}, X_{\mathrm{pt}}\right)=\left(P_{D} / W_{D}, X_{D}^{1}\right)
$$

be the Shimura datum underlying (the stratum containing) pt (Corollary 3.13) and $Q_{D} \supset P_{D}$ be the corresponding parabolic subgroup (see Proposition 3.3 and Lemma 3.8). Recall that $P_{D}$ and $Q_{D}$ have the same unipotent radical $W_{D}$ (Lemma 3.8).

By [8, 1.18] (see also [3, §2]), there are linear tensor functors

$$
\mu_{S}: \operatorname{Rep}_{G, \mathbb{Q}} \longrightarrow \operatorname{VHS}(S)_{\mathbb{Q}} \text { and } \mu_{\mathrm{pt}}: \operatorname{Rep}_{G_{\mathrm{pt}}, \mathbb{Q}} \longrightarrow \mathrm{HS}(\mathrm{pt})_{\mathbb{Q}},
$$

(called canonical construction functors) from the $\mathbb{Q}$-representations of $G$ (resp. $G_{\mathrm{pt}}$ ) to (admissible) variations of $\mathbb{Q}$-Hodge structures over $S$ (resp. over pt).

The functor $i^{*} j_{*}: \operatorname{VHS}(S)_{\mathbb{Q}} \longrightarrow \mathrm{HS}(\mathrm{pt})_{\mathbb{Q}}$ from (admissible) variations of $\mathbb{Q}$ Hodge structures over $S$ to $\mathbb{Q}$-Hodge structures over pt is left exact and, for any integer $k \geq 0$, we write

$$
R^{k} i^{*} j_{*}: \operatorname{VHS}(S)_{\mathbb{Q}} \longrightarrow \mathrm{HS}(\mathrm{pt})_{\mathbb{Q}}
$$

for the derived functor. Note that $R^{k} i^{*} j_{*}=i^{*} R^{k} j_{*}$.

Remark 4.2. Let $V$ be as in Notation 2.1 and let $f: A \longrightarrow S$ be the universal abelian scheme (Remark 2.7). Then one has a canonical identification

$$
\mu_{S}\left(V^{\vee}\right)=R^{1} f_{*} \mathbb{Q}_{A} .
$$


In particular, as $\mu_{S}$ is a tensor functor, all the relative cohomology sheaves of any $r$-fold fiber product of $A$ over $S$ are in the image of the functor $\mu_{S}$ (as well as several interesting direct factors of these sheaves, e.g. primitive parts with respect to a Lefschetz decomposition).

Lemma 4.3. The $\mathbb{Q}$-algebraic group $Q_{D} / W_{D}$ is isogenous to the direct product of a compact torus and $a \mathbb{Q}$-split torus. In particular, any neat arithmetic subgroup of $Q_{D} / W_{D}(\mathbb{Q})$ is trivial.

Proof. Consider the compact torus $T_{n}=\left\{(a, b), a^{2}+n b^{2}=1\right\}$, defined over $\mathbb{Q}$ (here $n$ is the integer of Notation 2.1). Then use the isogeny $\mathbb{G}_{m, \mathbb{Q}}^{2} \times T_{n}^{2} \longrightarrow Q / W_{1}$ given (using Convention 2.4) by

$$
\left(\lambda, \lambda^{\prime},(a, b),\left(a^{\prime}, b^{\prime}\right)\right) \mapsto\left(\begin{array}{ccc}
\lambda \lambda^{\prime}(a, b) & & \\
& \lambda^{\prime}\left(a^{\prime}, b^{\prime}\right) & \\
& & \lambda^{-1} \lambda^{\prime}(a, b)^{-1}
\end{array}\right) .
$$

Theorem 4.4. For any $F \in \operatorname{Rep}_{G}$, there is a canonical isomorphism of $\mathbb{Q}$-Hodge structures over $\mathrm{pt}$

$$
R^{k} i^{*} j_{*}\left(\mu_{S}(F)\right)=\mu_{\mathrm{pt}}\left(\left(H^{k}\left(W_{D}, F_{\mid Q_{D}}\right)\right)_{\mid G_{\mathrm{pt}}}\right),
$$

where $F_{\mid Q_{D}}$ is $F$ seen as representation of $Q_{D}$ and $H^{k}\left(W_{D}, \cdot\right)$ is the $k$-th derived of the functor that associates to a $Q_{D}$-representation its $W_{D}$-invariant part, and $\cdot_{\mid G_{\mathrm{pt}}}$ is again a restriction functor.

Proof. This is [3, Theorem 2.9] in a simplified version, that holds because the arithmetic group appearing in loc. cit. has to be trivial by Lemma 4.3 .

4.5. Notation. For any reductive group $H$, we will write $F_{\lambda, H}$ for the $H$-irreducible representation whose maximal weight is $\lambda$. We will simply write $F_{\lambda}$ if the group $H$ can be deduced from the context.

Theorem 4.6 (Kostant Theorem, see [12 thm 2.5.2.1). Let $H$ be a reductive group over $\mathbb{C}, B$ a Borel subgroup with unipotent radical $W$, $\Phi$ the associated root system, $\Phi^{+}$the subset of the positive ones, $\rho$ the half of the sum of positives roots, and $\mathcal{R}$ be the Weyl group. For any $\sigma \in \mathcal{R}$, define the length of $\sigma$ as $l(\sigma)=\#\left\{\alpha \in \Phi^{+}, \sigma^{-1} \alpha \notin \Phi^{+}\right\}$. Then one has an equality of $B / W$-representations $H^{k}\left(W, F_{\lambda, H}\right)=\oplus_{l(\sigma)=k} F_{\sigma(\lambda+\rho)-\rho, B / R}$

4.7. Lengths of roots. Let $\mathcal{B}$ be a parabolic basis adapted to $D$ (Definition 3.2). We have an isomorphism $\phi_{\mathcal{B}}: G_{\mathbb{C}} \stackrel{\sim}{\longrightarrow} \mathrm{GL}_{3, \mathbb{C}} \times \mathbb{G}_{m, \mathbb{C}}$ from Remark 2.3. Write $T_{s} \subset \mathrm{GL}_{3, \mathbb{C}}$ for the subgroup of upper-triangular matrices and $\Delta \subset \mathrm{GL}_{3, \mathbb{C}}$ for the diagonal ones. To describe the root system let us choose $\Delta \times \mathbb{G}_{m, \mathbb{C}} \cong \mathbb{G}_{m, \mathbb{C}}^{4}$ as maximal torus, and $T_{s} \times \mathbb{G}_{m, \mathbb{C}}$ as Borel subgroup containing the torus. Note that $\phi_{\mathcal{B}}$ restricts to an isomorphism: $\phi_{\mathcal{B}}: Q_{D, \mathbb{C}} \stackrel{\sim}{\longrightarrow} T_{s} \times \mathbb{G}_{m, \mathbb{C}}$. We write $\lambda_{1}, \ldots, \lambda_{4}$ for the four standard characters, which together form a basis for the lattice of characters. We also write $e_{i j}=\lambda_{i} \lambda_{j}^{-1}$. The simple roots are $e_{12}$ and $e_{23}$, the other positive root is $e_{13}$, and so $\rho=e_{13}$. 
The Weyl group $\mathcal{R}$ is the group of permutations of the first three coordinates of the characters. We will write elements of $\mathcal{R}$ with the standard notations for permutations; their lengths are given by

$$
l(e)=0, l(12)=l(23)=1, l(123)=l(132)=2, l(13)=3 .
$$

4.8. Computation. We keep notations from 4.7. Let $\lambda=(a, b, c, d)$ be any character written in the basis fixed in 4.7. Note that we have $\rho=(1,0,-1,0)$.

Let us compute $\sigma(\lambda+\rho)-\rho$ for all permutations $\sigma \in \mathcal{R}$.

$$
\begin{aligned}
e(a+1, b, c-1, d)-(1,0,-1,0) & =(a, b, c, d), \\
(12)(a+1, b, c-1, d)-(1,0,-1,0) & =(b-1, a+1, c, d), \\
(23)(a+1, b, c-1, d)-(1,0,-1,0) & =(a, c-1, b+1, d), \\
(13)(a+1, b, c-1, d)-(1,0,-1,0) & =(c-2, b, a+2, d), \\
(123)(a+1, b, c-1, d)-(1,0,-1,0) & =(c-2, a+1, b+1, d), \\
(132)(a+1, b, c-1, d)-(1,0,-1,0) & =(b-1, c-1, a+2, d) .
\end{aligned}
$$

By Theorem 4.6, we deduce the following equalities of $Q_{D, \mathbb{C}} / W_{D, \mathbb{C}}$-representations:

$$
\begin{aligned}
& H^{0}\left(W_{D, \mathbb{C}}, F_{\lambda, G_{\mathbb{C}} \mid Q_{D, \mathbb{C}}}\right)=F_{(a, b, c, d)}, \\
& H^{1}\left(W_{D, \mathbb{C}}, F_{\lambda, G_{\mathbb{C} \mid Q_{D, \mathbb{C}}}}=F_{(b-1, a+1, c, d)} \oplus F_{(a, c-1, b+1, d)},\right. \\
& H^{2}\left(W_{D, \mathbb{C}}, F_{\lambda, G_{\mathbb{C} \mid Q_{D, \mathbb{C}}}}=F_{(c-2, a+1, b+1, d)} \oplus F_{(b-1, c-1, a+2, d)},\right. \\
& H^{3}\left(W_{D, \mathbb{C}}, F_{\lambda, G_{\mathbb{C} \mid Q_{D, \mathbb{C}}}}=F_{(c-2, b, a+2, d)},\right.
\end{aligned}
$$

and $H^{k}\left(W_{D, \mathbb{C}}, F_{\lambda, G_{\mathbb{C}} \mid Q_{D, \mathbb{C}}}\right)=0$, for $k \geq 4$ (we are following Notation 4.5).

Remark 4.9. As $Q_{D, \mathbb{C}} / W_{D, \mathbb{C}}$ is isomorphic to the torus $\mathbb{G}_{m, \mathbb{C}}^{4}$ (see 4.7), the irreducible representations of $Q_{D, \mathbb{C}} / W_{D, \mathbb{C}}$ are just characters. In particular the six representations on the right hand side above are 1-dimensional and explicit.

4.10. Restriction to $\mathbb{S}$, the types. Consider $h_{\mathcal{B}, \infty}: \mathbb{S}_{\mathbb{C}} \rightarrow Q_{D, \mathbb{C}}$ of Definition 3.7 the induced map $\overline{h_{\mathcal{B}, \infty}}: \mathbb{S}_{\mathbb{C}} \rightarrow Q_{D, \mathbb{C}} / W_{D, \mathbb{C}}$, and the map $\phi_{\mathcal{B}}: Q_{D, \mathbb{C}} / W_{D, \mathbb{C}} \stackrel{\sim}{\longrightarrow} \mathbb{G}_{m, \mathbb{C}}^{4}$ defined in 4.7. The composition $\phi_{\mathcal{B}} \circ \overline{h_{\mathcal{B}, \infty}}: \mathbb{S}_{\mathbb{C}} \rightarrow \mathbb{G}_{m, \mathbb{C}}^{4}$. is then given by

$$
p h i_{\mathcal{B}} \circ \overline{h_{\mathcal{B}, \infty}}:\left(z_{1}, z_{2}\right) \mapsto\left(z_{1} z_{2}, z_{1}, 1, z_{1} z_{2}\right) .
$$

Hence, for any character $\lambda=(a, b, c, d)$ (written in the basis fixed in 4.7), one deduces from 4.8 the following equalities of $\mathbb{S}_{\mathbb{C}}$-representations

$$
\begin{aligned}
& H^{0}\left(W_{D, \mathbb{C}}, F_{\lambda, G_{\mathbb{C}} \mid Q_{D, \mathbb{C}}}\right)_{\mathbb{S}_{\mathbb{C}}}=F_{(a+b+d, a+d)}, \\
& H^{1}\left(W_{D, \mathbb{C}}, F_{\lambda, G_{\mathbb{C}} \mid Q_{D, \mathbb{C}}}\right)_{\mathbb{S}_{\mathbb{C}}}=F_{(a+b+d, b+d-1)} \oplus F_{(a+c+d-1, a+d)}, \\
& H^{2}\left(W_{D, \mathbb{C}}, F_{\lambda, G_{\mathbb{C}} \mid Q_{D, \mathbb{C}}}\right)_{\mathbb{S}_{\mathbb{C}}}=F_{(a+c+d-1, c+d-2)} \oplus F_{(b+c+d-2, b+d-1)}, \\
& H^{3}\left(W_{D, \mathbb{C}}, F_{\lambda, G_{\mathbb{C}} \mid Q_{D, \mathbb{C}}}\right)_{\mathbb{S}_{\mathbb{C}}}=F_{(b+c+d-2, c+d-2)}
\end{aligned}
$$

and $H^{k}\left(W_{D, \mathbb{C}}, F_{\lambda, G_{\mathbb{C}} \mid Q_{D, \mathbb{C}}}\right)_{\mathbb{S}_{\mathbb{C}}}=0$, for $k \geq 4$ (we are following Notation 4.5).

Theorem 4.11. Let $F_{\lambda}$ be the irreducible representation of $G_{\mathbb{C}}$ of maximal weight $\lambda=(a, b, c, d)$ (written in the basis fixed in 4.7). Then the following holds:

(1) $R^{0} i^{*} j_{*} \mu_{S, \mathbb{C}}\left(F_{\lambda}\right)$ has type $(-a-b-d,-a-d)$,

(2) $R^{1} i^{*} j_{*} \mu_{S, \mathbb{C}}\left(F_{\lambda}\right)$ has types $(-a-b-d,-b-d+1)$ and $(-a-c-d+1,-a-d)$,

(3) $R^{2} i^{*} j_{*} \mu_{S, \mathbb{C}}\left(F_{\lambda}\right)$ has types $(-a-c-d+1,-c-d+2)$ and $(-b-c-d+$ $2,-b-d+1)$, 
(4) $R^{3} i^{*} j_{*} \mu_{S, \mathbb{C}}\left(F_{\lambda}\right)$ has type $(-b-c-d+2,-c-d+2)$,

(5) $R^{k} i^{*} j_{*} \mu_{S, \mathbb{C}}\left(F_{\lambda}\right)$ vanishes for $k \geq 4$.

Proof. This is Theorem 4.4 with the computations done in 4.10

Remark 4.12. Note that the functor $R^{k} i^{*} j_{*} \mu_{S, \mathbb{C}}$ is linear, hence the types of $R^{k} i^{*} j_{*} \mu_{S, \mathbb{C}}(F)$ can be computed for any representation $F$ once we know its decomposition into irreducible factors.

Note also that all $\mathbb{C}$-representations $F_{\lambda}$ are defined over $E$ (as $G$ splits over $E$, see Remark 2.3), but not over $\mathbb{Q}$. If we start with a $\mathbb{Q}$-irreducible representation $F$, then $F_{E}$ will decompose in general in two factors, say $F_{E}=F^{1} \oplus F^{2}$ (e.g. $\left.V_{E}=F_{1,0,0,0} \oplus F_{0,0,-1,1}\right)$, and the types of $R^{k} i^{*} j_{*} \mu_{S, \mathbb{C}}\left(F_{\mathbb{C}}^{1} \oplus F_{\mathbb{C}}^{2}\right)$ will respect the Hodge symmetry.

Lemma 4.13. Let $p$ and $r$ be two non-negative integers, and let us keep notations

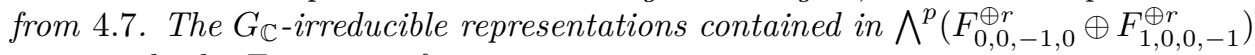
are exactly the $F_{a, b, c, d}$ verifying

(1) $r \geq a \geq b \geq c \geq-r$

(2) $3 r+a_{-}+b_{-}+c_{-} \geq-d \geq a_{+}+b_{+}+c_{+}$, and

(3) $a+b+c+2 d=-p$,

where, for any integer $x$, we define $x_{+}$(resp. $x_{-}$) as $x$ itself if it is positive (resp. if it is negative) and 0 otherwise.

Proof. In the representation $F_{0,0,-1,0}^{\oplus r} \oplus F_{1,0,0,-1}^{\oplus r}$ we have an explicit basis of $6 r$ elements that are eigenvectors for the action of the maximal torus. We deduce from them a basis $\mathcal{B}_{p}$ of $\bigwedge^{p}\left(F_{0,0,-1,0}^{\oplus r} \oplus F_{1,0,0,-1}^{\oplus r}\right)$ : each vector of $\mathcal{B}_{p}$ corresponds to the choice of $p$ between the $6 r$ previous elements. Then each vector of $\mathcal{B}_{p}$ is also an eigenvector for the action of the torus, whose weight is the sum of the weights of the $p$ elements chosen. From this we deduce that $a, b, c, d$ is a weight for the action of the maximal torus on $\bigwedge^{p}\left(F_{0,0,-1,0}^{\oplus r} \oplus F_{1,0,0,-1}^{\oplus r}\right)$ if and only if the integers verify

(1) $r \geq a, b, c \geq-r$,

(2) $3 r+a_{-}+b_{-}+c_{-} \geq-d \geq a_{+}+b_{+}+c_{+}$, and

(3) $a+b+c+2 d=-p$.

The condition $a \geq b \geq c$ corresponds to the choice of the Borel subgroup containing the maximal torus (as we did in \$4.7).

We need to show now that any $a, b, c, d$ verifying the condition in the statement is the maximal weight of a subrepresentation of $\bigwedge^{p}\left(F_{0,0,-1,0}^{\oplus r} \oplus F_{1,0,0,-1}^{\oplus r}\right)$. Suppose $-d \leq p / 2$ and $a, b, c$ negative (the other cases are analogous) and consider

$W_{a, b, c, d}=\left(\bigwedge^{3} F_{0,0,-1,0}\right)^{\otimes-a} \otimes\left(\bigwedge^{2} F_{0,0,-1,0}\right)^{\otimes a-b} \otimes\left(F_{0,0,-1,0}\right)^{\otimes b-c} \otimes\left(F_{0,0,0,-1}\right)^{\otimes-d}$.

The maximal weight of $W_{a, b, c, d}$ is $a, b, c, d$. To show that $W_{a, b, c, d}$ is a subrepresentation of $\bigwedge^{p}\left(F_{0,0,-1,0}^{\oplus r} \oplus F_{1,0,0,-1}^{\oplus r}\right)$ it is enough to show that $F_{0,0,0,-1}$ is a subrepresentation of $F_{0,0,-1,0} \oplus F_{1,0,0,-1}$. This is indeed the case: the action canonical paring of the standard representation of $\mathrm{GL}_{3}$ with its dual induces a non-zero morphism $F_{0,0,-1,0} \oplus F_{1,0,0,-1} \longrightarrow F_{0,0,0,-1}$. 
4.14. Proof of Theorem 1.3. First of all, we have the following identification

$$
R^{p} f_{*} \mathbb{Q}_{A^{r}}=\bigwedge^{p}\left(F_{0,0,-1,0}^{\oplus r} \oplus F_{1,0,0,-1}^{\oplus r}\right) ;
$$

see also Remark 4.2 and Remark 4.12 ,

We deduce from Theorem 4.11 and Lemma 4.13 that

(1) the $\mathbb{Q}$-Hodge structure $i^{*} R^{0} j_{*} R^{p} f_{*} \mathbb{Q}_{A^{r}}$ has weight $p-(a-c)$,

(2) the $\mathbb{Q}$-Hodge structure $i^{*} R^{1} j_{*} R^{p} f_{*} \mathbb{Q}_{A^{r}}$ has weights $p+1-(b-c)$ and $p+1-(a-b)$,

(3) the $\mathbb{Q}$-Hodge structure $i^{*} R^{2} j_{*} R^{p} f_{*} \mathbb{Q}_{A^{r}}$ has weights $p+3+(b-c)$ and $p+3+(a-b)$

(4) the $\mathbb{Q}$-Hodge structure $i^{*} R^{3} j_{*} R^{p} f_{*} \mathbb{Q}_{A^{r}}$ has weight $p+4+(a-c)$,

(5) the $\mathbb{Q}$-Hodge structure $i^{*} R^{k} j_{*} R^{p} f_{*} \mathbb{Q}_{A^{r}}$ vanishes for $k \geq 4$.

with $a, b, c$ varying between the numbers satisfying conditions in Lemma 4.13.

First of all, note that the map $(a, b, c, d) \mapsto(-c,-b,-a,-3 r-d)$ gives a bijection between the values of $(a, b, c, d)$ appearing in the list of irreducible subrepresentations of $\bigwedge^{p}\left(F_{0,0,-1,0}^{\oplus r} \oplus F_{1,0,0,-1}^{\oplus r}\right)$ and the values appearing in $\bigwedge^{6 r-p}\left(F_{0,0,-1,0}^{\oplus r} \oplus F_{1,0,0,-1}^{\oplus r}\right)$, so that we can suppose $p \leq 3 r$.

Note now that $a-b, b-c$ and $a-c$ are non-negative integers bounded by $2 r$ and $p$. Let us start by studying the possible values $t \leq p$ of $a-b$ (the case $b-c$ is similar). If $t \leq r$, then the triple $(a, b, c)=(t, 0,0)$ (or $(a, b, c)=(t, 0,-1)$, depending on the parity of $t$ ) verifies the conditions in Lemma 4.13 and gives $a-b=t$.

If $r<t \leq M_{p}$, then $(a, b, c)=(r, r-t, r-t)($ or $(a, b, c)=(r, r-t, r-t-1)$, depending on the parity of $t)$ verifies the conditions in Lemma 4.13 and gives $a-b=t$; it is also clear from this description that $a-b$ cannot take values bigger than $M_{p}$.

Let us now consider the possible values $t \leq p$ of $a-c$. If $0<t \leq r$, then the triple $(a, b, c)=(t, 0,0)$ (or $(a, b, c)=(t, 1,0)$, depending on the parity of $t$ ) verifies the conditions in Lemma 4.13 and gives $a-c=t$.

If $t=0$, then the triple $(a, b, c)=(0,0,0)$ if $p$ is even, or $(a, b, c)=(1,1,1)$ if $p$ is odd and at least 3 verifies the conditions in Lemma 4.13 and gives $a-c=0$; this description shows also that if $p=1$ then $a-c$ cannot be 0 .

If $r<t \leq C_{p}$, then $(a, b, c)=(r, 0, r-t)$ (or $(a, b, c)=(r, 1, r-t)$, depending on the parity of $t$ ) verifies the conditions in Lemma 4.13 and gives $a-c=t$.

4.15. Proof of Corollary 1.6. Let us show that $w=-a-b-c-2 d$. This equality and Theorem 4.11 clearly imply the statement.

Let us consider the morphism $p: \mathbb{G}_{m, \mathbb{C}} \longrightarrow \mathbb{S}_{\mathbb{C}}$ given by $z \mapsto(z, z)$, the morphism $h_{v_{1}, v_{2}, v_{3}}$ of Definition 2.5 and the morphism $\phi_{\mathcal{B}, \mathbb{C}}$ of Remark 2.3. The morphism $\phi_{\mathcal{B}, \mathbb{C}} \circ h_{v_{1}, v_{2}, v_{3}} \circ p: \mathbb{G}_{m, \mathbb{C}} \longrightarrow \mathrm{GL}_{3, \mathbb{C}} \times \mathbb{G}_{m, \mathbb{C}}$ is then given by $z \mapsto\left(z \cdot \mathrm{Id}, z^{2}\right)$. In particular the action of $z \in \mathbb{G}_{m, \mathbb{C}}(\mathbb{C})$ on $F_{(a, b, c, d)}$ via $\phi_{\mathcal{B}, \mathbb{C}} \circ h_{v_{1}, v_{2}, v_{3}} \circ p$ is the multiplication by $z^{a+b+c+2 d}$. By the very definition of the canonical construction [8, 1.18], this means that $w=-a-b-c-2 d$ (the sign comes from the convention $[8,1.3])$.

4.16. Acknowledgments. I thank Ishai Dan-Cohen, Javier Fresán, Marco Maculan, Siddarth Sankaran and Jörg Wildeshaus for helpful comments. I also thank 
the anonymous referee for helpful suggestions. I am grateful for the hospitality of the Hausdorff Research Institute for Mathematics, Bonn.

\section{REFERENCES}

[1] G. Ancona. Décomposition de motifs abéliens. Manuscripta Math., 146(3-4):307-328, 2015.

[2] J. Bellaïche. Congruences endoscopiques et représentations galoisiennes. PhD Thesis Univ. Orsay, 2002.

[3] J. Burgos and J. Wildeshaus. Hodge modules on Shimura varieties and their higher direct images in the Baily-Borel compactification. Ann. Sci. École Norm. Sup. (4), 37(3):363413, 2004.

[4] P. Deligne. Travaux de Shimura. In Séminaire Bourbaki, 23ème année (1970/71), Exp. No. 389, pages 123-165. Lecture Notes in Math., Vol. 244. Springer, Berlin, 1971.

[5] B. Gordon. Canonical models of Picard modular surfaces. In The zeta functions of Picard modular surfaces, pages 1-29. Univ. Montréal, Montreal, QC, 1992.

[6] M. Harris and S. Zucker. Boundary cohomology of Shimura varieties. III. Coherent cohomology on higher-rank boundary strata and applications to Hodge theory. Mém. Soc. Math. Fr. (N.S.), (85):vi+116, 2001.

[7] M. Larsen. Arithmetic compactification of some Shimura surfaces. In The zeta functions of Picard modular surfaces, pages 31-45. Univ. Montréal, Montreal, QC, 1992.

[8] R. Pink. Arithmetical compactification of mixed Shimura varieties. Bonner Mathematische Schriften [Bonn Mathematical Publications], 209. Universität Bonn Mathematisches Institut, Bonn, 1990. Dissertation, Rheinische Friedrich-Wilhelms-Universität Bonn, Bonn, 1989.

[9] A. Scholl. Motives for modular forms. Invent. Math., 100(2):419-430, 1990.

[10] J-P. Serre. Cours d'arithmétique. Presses Universitaires de France, Paris, 1977. Deuxième édition revue et corrigée, Le Mathématicien, No. 2.

[11] M. Stover. Cusps of Picard modular surfaces. Geom. Dedicata, 157:239-257, 2012.

[12] G. Warner. Harmonic analysis on semi-simple Lie groups. II. Springer-Verlag, New York, 1972. Die Grundlehren der mathematischen Wissenschaften, Band 189.

[13] J. Wildeshaus. Mixed sheaves on Shimura varieties and their higher direct images in toroidal compactifications. J. Algebraic Geom., 9(2):323-353, 2000.

[14] J. Wildeshaus. Chow motives without projectivity. Compos. Math., 145(5):1196-1226, 2009.

[15] J. Wildeshaus. Boundary motive, relative motives and extensions of motives. Panoramas et Synthèses. Autour des motifs. École d'été franco-asiatique de géométrie algébrique et de théorie des nombres. J.-B. Bost and J.-M. Fontaine eds., Vol. II:46 pages, 2011.

[16] J. Wildeshaus. On the interior motive of certain Shimura varieties: the case of HilbertBlumenthal varieties. International Mathematics Research Notices, 2012(10):2321-2355, 2012.

[17] J. Wildeshaus. On the interior motive of certain Shimura varieties: the case of Picard surfaces. Manuscripta Math., 148(3):351-377, 2015.

Universität Zurich, Winterthurerstr. 190, CH-8057 Zurich

E-mail address: giuseppe.ancona@math.uzh.ch

$U R L$ : http://user.math.uzh.ch/ancona/ 